\section{Robustecer protección de Comités Ético-Científicos}

\section{Reinforcing protection of Research Ethics Committees}

\section{Señor Editor:}

El excelente artículo de Portales et al. ${ }^{1}$ actualiza las normas éticas para investigación con seres humanos. Precisamente porque el aporte es una valiosa guía para Comités Ético-Científicos (CEC), conviene precisar algunos puntos que continúan siendo materia de debate, principalmente en lo referente a riesgos mínimos, definidos como "daños o molestias" que "los niños promedio-average-, sanos, normales, pueden enfrentar en su vida diaria o en experiencias con exámenes o pruebas psicológicas o físicas de rutina."2 . Este criterio es reconocido como excesivamente contextual, discrecional y variablemente interpretado por individuos y CECs, volviéndose aún más indeterminado desde que se amplia riesgos mínimos a "solo mínimamente aumentados" -minor increase-, con lo cual son adicionalmente inciertos por no haber criterios acordados sobre lo que es un mínimo aumento.

1) El concepto de riesgo mínimo ha sido elaborado para investigaciones biomédicas en niños y personas con competencia insuficiente para participar en el proceso de consentimiento informado previo a ingresar a un estudio que no tendrá beneficios directos para los probandos. Al ampliarse esta doctrina a estudio clínicos no terapéuticos, es decir, con niños enfermos que no beneficiarán médicamente de la investigación, se agregan dificultades de evaluación: los riesgos mínimos no son solo de la vida cotidiana de un niño sano, sino del enfermo que ya está en situación de riesgo aumentado por su afección y por lo efectos posibles de su tratamiento médico.

La doctrina de riesgo mínimo -con o sin riesgos incrementales menores- se refiere, por lo tanto, a estudios en niños y personas incapaces de consentir, y cuya inclusión en investigación que no los beneficiará proscribe que su consentimiento sea representado a menos que los riesgos sean mínimos. Que la situación sea contextual, discrecional, de estándares inciertos y variables, sugiere que los CEC no recurran a una revisión solo expedita. El recurso a la discrecionalidad de aplicar el concepto de riesgo mínimo y reducir el rigor de la evaluación de comité, es una estrategia que propende a la expedición evaluativa pero a costa de la máxima protección posible para probandos sobre todo infantiles. Conviene recordar la ya antigua regla de M. H. Pappworth, quien preguntaba al investigador acaso estaría dispuesto a someter a sus hijos al estudio que presenta.
2) La cita y reformulación del artículo de Emanuel, Wendler y Grady ${ }^{3}$ contiene algunas imprecisiones. Emanuel et al. presentan "7 requerimientos, todos necesarios, suficientes y universales", en tanto Portales et al. los reproducen como seis no coincidentes (la última erróneamente listada como a) en vez de f) y señalando que, según "propone Emanuel, una investigación tiene valor si cumple con algunas de estas características" (énfasis agregado para resaltar el desplazamiento de necesarios a contingentes). Publicado en el año 2000, el artículo del JAMA se refiere a valor social en forma genérica "generalizable a las poblaciones que utilizarán la intervención", una propuesta que es actualmente considerada insuficiente frente a la vaguedad del "valor social local" de investigaciones que se realizan en países de recursos reducidos -offshoring-, con aplicación en otras latitudes en incumplimiento del requerimiento que "el conocimiento buscado debe ser apropiadamente relevante para la comunidad huésped a objeto que la investigación sea ante todo ética"4. Este tema debiera ser prioritario para los CEC de nuestra región, considerando que muchos estudios aquí realizados ignoran las obligaciones de accesibilidad y disponibilidad local de lo investigado ${ }^{5}$.

Nuestros CEC deben estar atentos a la reducción de normativas de protección a probandos y naciones que hospedan investigaciones que no son localmente relevantes, una reducción que se hace sentir al leer que tanto la Declaración de Helsinki 2013, como las Guías CIOMS 2016, introducen comentarios discrecionales y proponen excepciones que debilitan garantías y compromisos de seguridad y protección a los investigados.

Dr. Miguel Kottow

Universidad Central de Chile y Universidad de Chile. Miguel.kottow@ucentral.cl

\section{Referencias}

1. Portales MA, Michaud P, Salas SP, Beca JP. Formas de Revisión Ética de Proyectos de Investigación Biomédica. Rev Med Chile 2017; 145: 386-92.

2. Wendler D, Emanuel EJ. What is a "minor" increase over minimal risk? J Pediatr 2005; 147: 575-8.

3. Emanuel EJ, Wenlder D, Grady C. What Makes Clinical Research Ethical? JAMA 2000; 283 (20): 2701-11.

4. Wenner DM. The Social Value of Knowledge and International Clinical Research. Dev World Bioeth 2015; 15 (2): 76-84.

5. Homedes N, Ugalde A. Availability and affordability of new medicines in Latin American countries where pivotal clinical trials were conducted. Bull World Health Organ 2015; 93 (10): 674-83. 